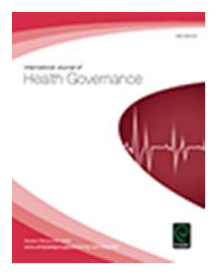

\title{
The pre-eminence of patient safety in health care
} governance

\begin{tabular}{|r|l|}
\hline Journal: & International Journal of Health Governance \\
\hline Manuscript ID & ijhg-12-2016-0054 \\
\hline Manuscript Type: & Clinical Governance Reviews \\
\hline Keywords: & $\begin{array}{l}\text { Clincal audit, Clinical governance, Culture, Health care quality, Patient } \\
\text { safety }\end{array}$ \\
\hline \multicolumn{2}{|l}{} \\
\hline
\end{tabular}

SCHOLARONE ${ }^{\text {Ix }}$

Manuscripts 
IJHG 22.1

\section{The pre-eminence of patient safety in health care governance}

In the field of health care, the first decades of the $21^{\text {st }}$ century will be remembered as the time period when patient safety became a central pillar of healthcare governance. This era was ushered in by the Institute of Medicine (IOM) report "To err is human: building a safer health system" (Kohn, Corrigan \& Donaldson 1999) which focused on the human cost of medical error. The report offered a clear and succinct definition of medical error as either the failure to complete a planned action, or as a planning failure where the action planned was unable to achieve the intended outcome. The authors concluded that medical error is divided into four major categories: diagnostic, treatment, preventative, other (Kohn, Corrigan \& Donaldson 1999). The category 'other' included anything that could not be filed under the first three categories such as systems failure or communication breakdown.

The importance of communication failure as a major contributor to medical error, and thus an environment in which patients are unsafe, was illustrated in a study that commenced in the same year that the IOM report was published (Sutcliffe, Lewton and Rosenthal 2004). The researchers interviewed a sample of twenty-six medical residents about incidents in which they had personally been involved and asked them to reflect on the underlying causes. In seventy cases of medical error, residents identified communication as a major cause. An important finding was that communication failure stems from hierarchical relationships, ambiguity about roles and responsibilities, and issues around conflict and power. In essence, communication error cannot be attributed entirely to the players directly involved, but is embedded in the institutional culture of the hospital (Sutcliffe, Lewton \& Rosenthal 2004).

Ten years later, communication failure continued to be a major cause of medical error, leading to a shortfall in patient safety. A dangerous trigger point for communication error causing harm to patients was identified as the 'hand off', or period of time when the care of a patient is transferred from one health care practitioner to another (Starmer, Spector, Srivastava et al 2014). In a prospective study published by the prestigious New England Journal of Medicine, the authors introduced a package, the I-Pass Handoff Bundle, (Starmer, Spector, Srivastava et al 2014) which included a mnemonic to use during medical handover, protocols for staff training and suggestions for faculty development. When researchers evaluated changes using pre and post intervention statistics, preventable errors at six out of the nine research sites decreased by 30\%. This improvement reflects not only an acquired skill, but also a cultural shift within the institutions themselves.

Ferlie and Shortell (2001) recognised the necessity of transforming institutional culture in order to improve patient safety in both the UK and the USA.

Institutional culture is sometimes intangible, they note, but always complex and a fundamental factor when altering operational norms. A culture that supports innovation and inter-professional dialogue can act as a powerful catalyst for 
change. Conversely, a closed, rigidly hierarchical culture can build barriers. A major source of conflict is often between the clinical culture and the managerial culture of an institution (Ferlie and Shortell 2001). A substantial change in this relationship occurred in 1999 in the UK as NHS Trusts became legally responsible for patient safety as part of the emerging clinical governance agenda (Flynn 2002). This was reinforced by the introduction of the National Patient Safety Agenda (NPSA) in 2001, with the stated goal of reducing harm to patients (Emslie, Knox and Pickstone 2002). While acknowledging that in a large organisation such as the NHS, mistakes will occur, the NPSA sought to change the institutional culture of the NHS from a blame culture to a learning culture in which mistakes can be acknowledged, analysed, reflected upon and learned from (Emslie, Knox and Pickstone 2002). Since this time, clinical governance has become the keystone of quality enhancement and evaluation within the NHS. In essence everything that contributes to an improvement in health care shelters under the clinical governance umbrella (Brennan and Flynn 2013). This includes audit, research, national standards, evidence-based practice, incident reporting, continuing professional development and risk management (Braithwaite and Travaglia (2008).

This issue of IJHG presents a variety of articles illustrating how the overarching aim of good healthcare governance, to alter the culture of care in order to improve patient safety, is played out in different settings. From the new national health workforce regulation in Australia (Pacey, Smith-Merry, Gillespie and Short 2017), through the introduction of a clinical governance framework in a medical combat unit (Currie, Mateer, Weston et al 2017) and an initiative to make hospital wards safer for patients with dementia (Eastham and Cox 2017) to a regional-based collaboration to increase patient safety (Veit 2017), all the articles have a common theme of improving patient safety through changes in the existing health care culture. Finally, Wood, Shaw, Sivananthan et al (2017) continue this theme with a review of a restraint technique used in psychiatric facilities, while Berland (2017) explores the use of the Johari Window to illustrate the interaction and divergence of patient and provider perspectives in health care encounters. Understanding and valuing the patient perspective is another example of how changing a healthcare culture from a "doctor knows best" model to one where the views and concerns of patients contribute to a collaborative approach to care, improves both patient safety and satisfaction.

Pacey et al (2017) illustrate the changing culture of Australian healthcare regulation as it moved, in 2010, from a patchwork of territorial, state or commonwealth regulations to a unified system embodied in a national framework for health legislation. This has contributed to patient safety by ensuring that standards are the same throughout Australia. Prior to 2010 state regulation of health professionals meant that in some professions, licensure was recognized in some states but not in others. This prohibited easy movement of health care workers, required to meet the demands of changing demographics, particularly the challenges associated with a rapidly ageing population. Pacey et al (2017) point out that although a national framework for health care regulation has many benefits, this may, in some cases, challenge the ability of states to regulate health care in response to the specific needs of their own populations. 
This is somewhat compensated by the establishment of 137 local hospital networks (LHNs) which consist of small groups of public hospitals. LHNs have a responsibility for service delivery, outcomes, and budget management; they are therefore able to respond to local needs (Schweppenstedde, Hinrichs, Ogbu et al 2014). The overall consensus is, nevertheless, that the regulatory framework should, in the long term, demonstrate benefits in terms of patient safety on a national level. However, as this is still an evolving project, comprehensive audit data sets may not yet be available. For example, Australian National Safety and Quality Health Service (NSQHS) standards published in 2011 by the Australian Commission on Safety and Quality in Health Care (ACSQHC) were only adopted nationally in 2013. These standards cover ten essential areas of health care relating to patient safety with a view toward establishing procedures for dealing with adverse incidents (Schweppenstedde, Hinrichs, Ogbu et al 2014).

Another example of the changing culture of health care is presented by Currie, Mateer, Weston et al (2017). The authors report on the development of a clinical governance framework designed to improve patient safety within Australian deployed military units. One challenge identified is the cultural dissonance between the military tradition of obedience and the joint responsibility of health practitioners at all levels which provides checks and balances within a clinical governance framework.

This however can be viewed as a learning opportunity, which is not entirely onesided. A great deal of knowledge in acute medicine and surgery, particularly in trauma care, is derived from experiences and innovations in caring for soldiers wounded in combat. A clinical governance framework affords the opportunity to provide education about best practice standards, using knowledge gained in the field. An example is the standardisation of resuscitation procedures for patients requiring massive blood transfusions. Following the adoption of an evidencebased guideline across all military units, the percentage of soldiers surviving following massive haemorrhage increased by over $10 \%$.

While improving soldiers' ability to survive injuries sustained in battle is a noteworthy goal, it is important to realise that for some people, the activities of daily living are paramount to a continuous battle. This is especially true for patients with dementia. Confusion can be exacerbated for people with dementia by unfamiliar surroundings, thus environment has been increasingly recognised during the past decade as a therapeutic resource in the care of people with Alzheimers and other forms of dementia (Day, Carreon and Stump 2000). Eastham and Cox (2017) report on a literature review and a pilot study of patient engagement in the activities of daily living (Holland 2008) while living with a diagnosis of dementia. The authors describe the features of a dementia friendly environment, as endorsed by the Kings Fund, as "familiarity, legibility, wayfinding, orientation and daily activity engagement". The Kings Fund developed an assessment tool together with a number of NHS trusts. The enhancing the healing environment (EHE) tool contains seven criteria, which are scored independently to assess whether a ward can be considered 'dementia friendly' (Kings Fund 2014). Eastham and Cox used the criteria from the Kings Fund to design a ward environment and then assessed patient engagement and 
interactions with staff. They found that simple geometric shapes, warm contrasting colours and a bright environment were beneficial for dementia patients. The authors acknowledged the limitations of the study due to a small sample size and the acute hospital setting. However, the setting may not have been the limitation that the researchers assumed it to be. In an earlier review of the literature, Day, Carreon and Stump (2000) found that a home-like setting within a clinical facility was not necessarily more beneficial for dementia patients, in terms of patient safety and engagement, than a more traditional ward environment. This is undoubtedly an important area of both research and governance in view of increasing lifespans, resulting in a rising number of people diagnosed with dementia.

Veit (2017) reports on a more strategic approach to increasing patient safety by reducing disparities in health care in Washington State. Established in the early years of the $21^{\text {st }}$ century, the Washington Patient Safety Coalition (WPSC) was formed when the Washington state Department of Health (DOH) and the Health Care Authority (HCA) approached the Foundation for Health Care Quality (FHCQ) to create a working group for the purpose of addressing safety issues.

The WPSC's role is to create a safe space where members can discuss and debate patient safety issues. In addition, the WPSC acts as an education hub where resources can be shared, educational campaigns are planned, and an annual conference is organised.

The WPSC is led by a steering committee comprised of a rotating membership of 15 , made up of representatives from the thirty-eight organisations represented. A second tier of governance is the advisory committee, which is made up of one representative from each organisation. While members of the advisory committee can attend steering committee meetings, only steering committee members have a vote when deciding WPSC business.

The WPSC takes an active role in matters of patient safety by providing links to external information sources, hosting monthly webinars, holding an annual conference, and creating campaigns about specific safety issues. A recent campaign addressed medication safety. In addition, the WPSC works closely with other agencies such as the Washington Health Alliance and the Governor's Interagency Council to reduce health disparity and improve patient safety in the state of Washington.

Wood, Shaw and Sivananthan et al's (2017) article returns us to a specific issue with great importance to patient safety: the use of restraint with psychiatric patients. There can be no greater aim in the field of patient safety than preventing unnecessary death. Restraining a vulnerable individual in a facedown position (prone restraint) can lead to severe trauma, or even death by impairing a patient's airway or reducing circulation. Wood, Shaw and Sivananthan et al (2017) report on an audit of prone restraint, a controversial technique, which some mental health charities and organisations say should be banned. Some NHS trusts such as Cheshire and Wirral Partnerships NHS Foundation Trust (CWP), have already discontinued authorisation of prone 
restraint. Nevertheless, in the this trust, its use has actually increased since 2013 to 284 instances per 100,000 bed days and in over $40 \%$ of all incidents involving restraint, prone restraint was used. Data from the audit demonstrated that in $93 \%$ of all incidents ending in the use of prone restraint, staff attempted to calm the patient by using non-restrictive techniques before employing the controversial restraining method. Findings from the audit indicated that patients subjected to this type of restraint had most commonly been diagnosed with paranoid schizophrenia or unstable personality disorder. Questions asked during the data collection phase included information about who was restrained (age, gender and diagnosis), why the restraint occurred, where it happened and how long it lasted. The audit showed that only $40 \%$ of prone restraining incidents were followed up by a staff debriefing session, and where these did occur, they were undocumented. Patient reviews took place in over half of the incidents but where these occurred, patient care plans were not updated.

This audit demonstrated that even in NHS trusts where the commitment to discontinue dangerous practices exists, prone restraint was still being used at the time of this audit with inadequate follow-up or documentation. The good news is that the audit process enabled staff to see what was happening and review their own actions, and those of their colleagues. At the time of writing this article, the authors report that the most recent audit results show that CWP now has reduced the use of prone restraint to less than the national average number on adult acute wards, psychiatric intensive care units and high dependency inpatient services. Information from patients on the use of this technique would have provided additional insights, which might be used to reduce even further, or even eliminate prone restraint entirely.

The final article reviewed focuses on just such patient perspectives through the use of the Johari Window. Psychologists Harrington (Harry) Ingham and Joseph Luff developed the Johari Window in 1955 (Armstrong 2006). While the name sounds exotic to Anglophones, it is simply a combination of the names of its creators (Joe \& Harry). Designed as a tool to promote self-understanding and interpersonal communication, its descriptors includes 'soft' attributes such as helpfulness, sympathy and kindness. Berland (2017) reports on the use of the Johari Window to explore patient and provider perspectives. This tool was selected because of the way it presents a visual representation of information. The window is divided into four quadrants. One quadrant represents knowledge that the patient alone has, while its corresponding window contains knowledge known only to the care provider. The third window represents information shared by both the patient and the provider, while the final window depicts information known to neither of them.

While Berland (2017) acknowledges that there has been no formal evaluation of using this tool in health care planning, it has been used more widely in other fields where it has evaluated well (Armstrong 2006). It may, however, be particularly useful in health care where knowledge tends to be unevenly distributed among the various players. For instance, a surgeon may have a precise understanding of his or her surgical procedure: knowledge, which is not held by the patient. However the surgeon may have little understanding of what 
it feels to be a patient who is fearful of dying on the operating table. In fact, this information may be hidden to the patient's conscious mind but manifest itself as anxiety or depression prior to surgery.

While Canadian hospitals often use 'scorecards' or 'dashboards' to summarise clinical quality indicators (CQI), these are often missing the patient perspective. When patient data is collected it is often superficial and does not capture patients' true views or deepest feelings about their hospital in-patient experience (Berland 2017). An example is patients who rate their hospital stay highly on discharge surveys, yet can talk to researchers about specific problems such as poor discharge planning.

Another benefit of the Johari Window is that it can accommodate both qualitative and quantitative data. This provides a more comprehensive picture of what works and what doesn't in health care and can aid in improvement planning (Berland 2017).

Patient safety continues to occupy the minds of both patients and of health care providers. The latter seek to enhance governance strategies to stimulate continual improvement in patient safety and satisfaction. The former consider whether they or their loved ones will be safe when entering a health care facility. Questions about infection control, surgical risk, being treated with dignity and respect, risk management and medication errors are asked on both sides of the surgical table or hospital bed. These are questions that must continue to be asked, and solutions found, to ensure patients remain safe and health practitioners can do their jobs with the assurance that they will be able to fulfill the edict of the Hippocratic oath, to do no harm.

\section{References:}

Armstrong T (2006) Revisiting the Johari Window: Improving communications through self-disclosure and feedback, Human Development 27(2): 10

Berland A (2017) Using the Johari Window to explore patient and provider perspectives, IJHG 22(1)

Braithwaite J and Travaglia JF (2008) An overview of clinical governance policies, practices and initiatives, Australian Health Review 32(1): 10-22

Brennan, NM and Flynn, MA (2013) Differentiating clinical governance, clinical management and clinical practice, Clinical Governance: An International Journal, 18(2):114-131

Currie J, Mateer J, Weston D, Anderson E, Harding J (2017) Implementation of a clinical governance framework to 17 Combat Service Support Brigade, Australian army, IJHG 22(1) 
Day K, Carreon D, Stump C (2000) The therapeutic design of environments for people with dementia: A review of the empirical research The Gerentologist 40(4): 397-416

Eastham A and Cox D (2017) Dementia-friendly wards: a review of the literature and pilot study of patient interaction and daily activity engagement, IJHG 22(1)

Emslie S, Knox C, Pickstone M (Eds.) (2002) Improving Patient Safety: Insights From American, Australian and British Healthcare London: Department of Health

Ferlie EB and Shortell SM (2001) Improving the quality of healthcare in the United Kingdom and the United States: A framework for change, The Milbank Quarterly 79(2): 281-315

Flynn R (2002) Clinical governance and governmentality. Health, Risk and Society 4(2): 155-173

Holland K (Ed.)(2008) Applying the Roper-Logan-Tierney Model in Practice. $\left(2^{\text {nd }}\right.$ edition) Amsterdam: Elsevier Health Sciences

Kohn LT, Corrigan JM, Donaldson MS (Eds.) (1999) To Err is Human: Building a Safer Health System. Report by the Committee on Quality of Health Care in America, IOM. Washington D.C: National Academy Press

Pacey F, Smith-Merry J, Gillespie J, Short S (2017) National health workforce regulation: Contextualising the Australian scheme, IJHG 22(1)

Schweppenstedde D, Hinrichs S, Ogbu UC, Schneider EC, Kringos DS, Klazinga NS, Healy J, Vuorenkoski L, Busse R, Guerin B, Pitchforth E, Nolte E (2014) Regulating quality and safety of health and social care. International experiences. Washington \& Canberra: Rand Corporation

Starmer AJ, Spector ND, Srivastava R et al (2014) Changes in medical errors after implementation of a handoff program, New England Journal of Medicine 371:1803-1812

Sutcliffe KM, Lewton E, Rosenthal M (2004) Communication failures: An insidious contributor to medical mishaps, Academic Medicine 79(2): 186-194

The Kings Fund (2014) Is Your Ward Dementia Friendly? EHE Environmental Assessment Tool. Accessed on 11.12.2016 from:

https://www.kingsfund.org.uk/sites/files/kf/EHE-dementia-assessmenttool.pdf

Veit K (2017) Regional patient safety and quality leaders aim to reduce disparities in healthcare with collaborative approach, IJHG 22(1) 
International Journal of Health Governance

Page 8 of 8

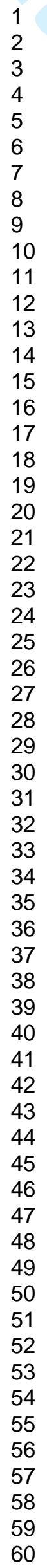

Wood D, Shaw E, Sivananthan A, Partington J, Reave A, Fishwick H (2017) Use of prone position restraint within a mental health trust: A clinical audit of psychiatric practice and methods for improvement, IJHG 22(1) 